\title{
Structure analysis of subjective well-being
}

\author{
Leopoldo Daniel-González, ${ }^{\prime}$ José Moral de la Rubia, ${ }^{1}$ Adrián Valle de la O, ${ }^{2}$ Cirilo H. García-Cadena'
}

Facultad de Psicología, Universidad Autónoma de Nuevo León, Monterrey, México.

2 Escuela de Medicina y Ciencias de la Salud, Instituto Tecnológico y de Estudios Superiores de Monterrey, Monterrey, México.

\section{Correspondence:}

Leopoldo Daniel González

Facultad de Psicología, Universidad

Autónoma de Nuevo León.

Dr. Carlos Canseco 110 ,

Col. Mitras Centro,

C.P. 64460, Monterrey, N. L.,

México.

Phone: (81) 8333 - 8233

Email: leopoldo.danielgn@uanl.edu.

$\mathrm{mx}$

Received: 22 November 2019

Accepted: 23 March 2020

Citation:

Daniel-González, L., Moral de la Rubia, J., Valle de la O., A., \& García-Cadena, C. H. (2020). Structure analysis of subjective well-being. Salud Mental, 43(3), 1 19-127.

DOI: 10.1771 1/SM.0185-3325.2020.017

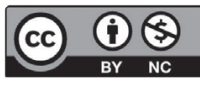

\begin{abstract}
Introduction. There is controversy regarding the structure of subjective well-being (SWB) and the possibility of calculating a SWB total score. Objective. To test and compare five models proposed for the description of SWB. Method. The study was implemented with a cross-sectional, ex-post-facto design using an incidental sampling method. The Positive and Negative Experience Scale and the Satisfaction with Life Scale were applied to a sample composed of 600 students of health sciences from two universities of Nuevo Leon, Mexico. Data were analyzed through a structural equation modeling, using Maximum Likelihood and Corrected-Bias Percentile methods. Results. The bifactor model comprising three specific factors, vis-à-vis the model composed of three correlated factors, had the best data fit $\left(\Delta \chi^{2} / \Delta d f=8.166>5, \Delta N F I=.018, \Delta N N F I=.015\right.$, and $\Delta C F I=.016>.01)$, and all its fit indices were close; however, the specific factor related to positive affect had a poor contribution. Nevertheless, the model composed of three correlated factors had the greatest parsimony $(P R=.853, P N F I=.804, P N N F I=.813, P C F I=.819$, and $P G F I=.706)$ and its three factors showed convergent validity, discriminant validity, and internal consistency reliability. Discussion and conclusion. The two models with the best properties justify the use of a composite score of SWB based on the scores of positive affect, negative affect, and satisfaction with life, as well as scores for these three specific domains of content. From a psychometric perspective, the model composed of three correlated factors yielded the best result.
\end{abstract}

Keywords: Subjective well-being, personal satisfaction, affect, psychometrics, latent class analysis, students.

\section{RESUMEN}

Introducción. Hay una controversia en torno a la estructura del bienestar subjetivo (BS) y la posibilidad de calcular una puntuación total del BS. Objetivo. Contrastar y comparar cinco modelos propuestos para el BS. Método. El diseño del estudio fue ex-post-facto de corte transversal. Se usó un muestreo incidental. La Escala de Experiencias Positivas y Negativas y la Escala de Satisfacción con la Vida se aplicaron a una muestra compuesta por 600 estudiantes de ciencias de la salud de dos universidades de Nuevo León, México. Los datos se analizaron por modelamiento de ecuaciones estructurales, usando Máxima Verosimilitud y Percentiles Corregidos de Sesgo. Resultados. El modelo bifactor de tres factores específicos tuvo, respecto del modelo compuesto por tres factores correlacionados, tuvo el mejor ajuste a los datos $\left(\Delta \chi^{2} / \Delta d f=8.166>\right.$ $5, \Delta N F I=.018, \Delta N N F I=.015$ y $\triangle C F I=.016>.01)$ y todos sus índices de ajuste fueron buenos; no obstante, el factor específico de afecto positivo tuvo una contribución pobre. Sin embargo, el modelo de tres factores correlacionados tuvo la mayor parsimonia $(P R=.853, P N F I=.804, P N N F I=.813, P C F I=.819$ y PGFI $=.706)$ y sus tres factores mostraron validez convergente, validez discriminante y consistencia interna. Discusión y conclusión. Los dos modelos con mejores propiedades justifican el uso de una puntuación compuesta de BS integrada por afecto positivo, afecto negativo y satisfacción con la vida, así como puntuaciones para estos tres dominios específicos de contenido. Desde la perspectiva psicométrica, el modelo de tres factores correlacionados proporcionó el mejor resultado.

Palabras clave: Bienestar subjetivo, satisfacción personal, afecto, psicometría, análisis de variables latentes, estudiantes. 


\section{INTRODUCTION}

Since its emergence in the 1960s, the conceptualization of subjective well-being (SWB) has been an important endeavor for psychology (Maddux, 2018). The study of SWB has grown tremendously in the last 30 years, and increasingly complex methodologies are being used for its measurement, as well as for the study of its effects upon individual adaptation, culture, and personality (Diener et al., 2017).

SWB is generally defined as the assessment that people make, in cognitive and affective terms about their own lives. Thus, it can be said that SWB has three components: life satisfaction (LS), which refers to the explicit and conscious evaluations that individuals do regarding their own life, positive affect (PA), which refers to pleasant and desirable emotional feelings and moods, and negative affect (NA), which refers to unpleasant and undesirable emotional feelings and moods (Diener, Lucas, \& Oishi, 2018). Since this tripartite model was proposed (Diener, 1984), it has become the most used; nevertheless, it has been questioned because it does not represent a unitary assessment of SWB (Quezada, Landero, \& Gonzalez, 2016). Furthermore, since not enough empirical evidence exists to support the tripartite model, a great controversy has emerged with regard to the possible relationship between its cognitive component and its two affective components (Jovanović, 2015).

Notwithstanding the enormous progress made in the field of SWB, there are still unresolved issues concerning its factorial structure. Upon performing a search in the scientific literature, Busseri and Sadava (2011) identified five models that attempt to conceptualize the study of SWB in order to more fully understand its structure, measurement, analysis, interpretation, and composition. These five models are as follows: one composed by three separate components; a hierarchical model composed by three components and a higher order factor; a causal system model in which affectivity is seen as an important input for attaining LS; a composite model in which SWB represents a composite experience comprising LS, NA, and PA; and finally a model in which SWB is conceptualized in terms of a distinct configuration of components that leads each individual to experience SWB.

The five aforementioned models have limitations that do not allow for a full understanding of SWB. The model composed of three separate components cannot explain the existence of common sources of variance in SWB. In the hierarchical model, the general and specific influences upon the observed indicators cannot be evaluated simultaneously. In the causal system model, SWB is not clearly framed, and LS is explained by an opposite sign effect due to PA and NA. In the composite model, SWB presents its own measurement model and is structurally determined by LS, PA, and NA, which is considered conceptually inappropriate. The components configuration model, more than an explanation of the construct, represents a classification of individuals into different patterns of LS, PA, and NA (Busseri \& Sadava, 2011). Given these inadequacies, Jovanović (2015) has proposed a bifactor model comprising three specific factors (LS, PA, and NA) to conceptualize SWB. In that study, the bifactor model better explains the variance of the set of items than the models composed of one, two, and three factors, regardless of whether the factors are independent, correlated, or hierarchized to a general factor.

The bifactor model is made up of a general factor and a certain number of specific factors, so that the variance of each item is explained by both the general factor and a specific factor. This model specifies that the correlations between items can be accounted for by a general factor (shared variance between all items) and a series of specific factors (shared variation between the items belonging to a specific domain of content). The use of this type of models allows the understanding of multidimensional structures and, in turn, allows determining what scores can be reliably assessed (Reise, Bonifay, \& Haviland, 2018).

Few researches have focused on analyzing the structure of the SWB from its three components; four studies doing so are the one conducted by Rodríguez-Fernández and Goñi-Grandmontagne (2011), another one by Metler and Busseri (2015), and the studies conducted by Busseri $(2015,2018)$. There is even less research that has considered the bifactor model. Three recent studies, conducted by Chen, Jing, Hayes, and Lee (2013), Jovanović (2015), and Lapuente, Dominguez-Lara, Flores-Kanter, and Medrano (2018), used the Satisfaction with Life Scale (SWLS) (Diener, Emmons, Larsen, \& Griffin, 1985) to assess the cognitive component of SWB, as well as the Positive and Negative Affect Schedule (PANAS) (Watson, Clark, \& Tellegen, 1988) to assess the affective component of SWB. Nevertheless, it is known that the correlation between the affective factors in PANAS ranges from weak to moderate (Seib-Pfeifer, Pugnaghi, Beauducel, \& Leue, 2017; Moral, 2019a), which could be considered as a limitation for the use of the bifactor model because strong associations between the specific factors are required in order to hypothesize the existence of a general factor (Reise et al., 2018). Thus, the Scale of Positive and Negative Experience (SPANE) (Diener et al., 2010), whose two affective factors have consistently yielded strong to very strong correlation coefficients (Rahm, Heise, \& Schuldt, 2017), is a better option. Therefore, we proceeded to analyze the structure of SWB considering the bifactor model comprising three specific factors, using SWLS to assess LS and SPANE to assess affects. It should be mentioned that SPANE has not been previously used in the study of the structure of the SWB.

The aim of this study is to find out which one of the five models intended to explain SWB is the best for representing this construct. The bifactor model comprising three specific factors is expected to be the best. 


\section{METHOD}

\section{Study design}

The study was implemented with a cross-sectional, ex-postfacto design using an incidental sampling method.

\section{Participants}

The participants were recruited from two universities from the state of Nuevo Leon. Since the sampling procedure was non-probabilistic, the sample size was determined by the rule of thumb of having at least 400 participants to use a confirmatory factor analysis and a minimum of 10 participants per parameter to estimate. The sample size was composed by 600 participants, with which these minimums were reached: 16.2 participants per parameter to estimate in the one-factor model; 15.8 in the two-correlated factor model; 11.1 in the bifactor model with two specific factors; 15 in the three-correlated factor model; and 11.1 in the bifactor model with three specific factors. It should be noted that the minimum sample size to calculate the three-factor model with 17 observed variables under the assumption of multivariate normality with a power of .80 and a significance level of .05 is 156 participants (Westland, 2010). This calculation of a priori sample size was done using the Soper's software (2020).

\section{Measurement}

The questionnaire used in this study comprised questions regarding socio-demographic information and two Likerttype, self-report scales:

1. Scale of Positive and Negative Experience (Diener et al., 2010). This is a self-report scale composed of 12 items related to affect: 6 items for PA and 6 items for NA. The items are rated along a five-point, Likert-type scale (from 1 = "never" to 5 = "always"). This study used the Spanish language version developed by Daniel-González, Moral de la Rubia, Valle de la O., García-Cadena, and Martínez-Martí (2019). In this study, the reliability coefficients of the two factors composing this scale were excellent for PA $(\omega=.93)$ and good for NA $(\omega=.87)$.

2. Satisfaction with Life Scale (Diener et al., 1985). This is a self-report scale composed of five items that are usually rated along a seven-point, Likerttype scale (from 1 = "totally disagree" to 7 = "totally agree"). The scale has a good internal consistency $(\alpha=.88)$. This study used the Spanish language version developed by Vázquez, Duque, and Hervas (2013). In this study, the reliability coefficient was good $(\omega=.86)$.

\section{Procedure}

Before applying the tests, permission was requested from the corresponding academic authorities of each university. Students were invited to participate voluntarily, signing the corresponding informed consent. After explaining the objective of the study and giving the pertinent instructions, the questionnaire was applied in the classrooms. Respondents received no economic, material, or academic compensation for participating in this study.

\section{Data analysis}

Statistical analyses were performed through AMOS24 and SPSS24. To test the models, confirmatory factor analysis was used. The discrepancy function was optimized by the maximum likelihood method. The 95\% confidence intervals for parameters and two-tailed significance tests were calculated using bias-corrected percentile method with 2,000 bootstrap samples.

The goodness of fit was assessed through eight indices: relative chi-square $\left(\chi^{2} / d f\right)$, Goodness-of-Fit Index $(G F I)$, Adjusted Goodness-of-Fit Index $(A G F I)$, Normed Fit Index (NFI), Non-Normed Fit Index (NNFI), Comparative Fit Index $(C F I)$, Standardized Root Mean Square Residual $(S R M R)$, and Root Mean Square Error of Approximation (RMSEA) with its point estimate and 90\% confidence interval. The criteria to establish that the proposed models showed a close fit were: $\chi^{2} d f \leq 2$; GFI, NFI, NNFI, and $C F I \geq .95 ; A G F I \geq .90$; and $S R M R$ as well as $R M S E A \leq .05$. Indices values considered as indicating an adequate goodness of fit were: $\chi^{2} / d f \leq 3$; GFI, NFI, NNFI, and $C F I \geq .90$; $A G F I \geq .85 ; S R M R \leq .10$; and $R M S E A \leq .08$. The equivalence in goodness of fit between two models was tested through the chi-square difference test, relative chi-square difference $\left(\Delta \chi^{2} / \Delta d f\right)$, and the differences in the indices $G F I$, $N F I, A G F I$, and $C F I$. A $p$-value $>.05$ for the null hypothesis of equivalence $\left(\mathrm{H}_{0}: \Delta \chi^{2}=0\right), \Delta \chi^{2} / \Delta d f<2$, and $\Delta C F I, \Delta N F I$, and $\triangle N N F I \leq .01$ were considered to show an equivalence in goodness of fit (Byrne, 2016).

The parsimony of each model was measured through the parsimony ratio $(P R)$. A $P R$ value $<.20$ was interpreted as a very low parsimony, from .20 to .39 , low, from .40 to .59 , medium, from .60 to .79 , high, and from .80 to 1 , very high. The parsimony fit indices (PGFI, PNFI, PNNFI, and $P C F I$ ) were also calculated. $P G F I$ values $\geq .70$ and $P N F I$, $P N N F I$, and $P C F I \geq .80$ are considered as indicating a good relation between fit and parsimony, whereas $P G F I \geq .50$ and $P N F I, P N N F I$, and $P C F I \geq .60$ are considered as indicating an acceptable relation (Byrne, 2016).

In the one-factor model and in the two-correlated factor model, the composite reliability was calculated through McDonald's Omega coefficient and construct reliability through Hancock-Muller's $H$ coefficient. Values of $\omega$ and $H$ 
between .70 and .79 represent acceptable reliability, between .80 to .89 good, and $\geq .90$ excellent. Convergent validity was tested by the average variance extracted $(A V E>.50)$. In the two-correlated factor model, the discriminant validity between pairs of factors was verified by a shared variance lower than the $A V E$ of each factor (Moral, 2019b).

In the bifactor model, the contribution of each factor (specific and general) to each content domain was evaluated through ten indices: the McDonald hierarchical omega related to the specific factor $\left[\omega_{\mathrm{h}(\mathrm{SF})}\right]$ and the general factor $\left[\omega_{\mathrm{h}(\mathrm{GF})}\right]$, the average of the common variance of the items explained by the specific factor $\left[\mathrm{M}_{\left(\mathrm{ECV} \_ \text {I_SF }\right)}\right]$ and the general factor $\left[\mathrm{M}_{\left(\mathrm{ECV} \_ \text {I_GF }\right.}\right]$, the common variance explained by the specific factor $\left(E C V \_S F\right)$ and the general factor $\left(E C V \_G F\right)$, the average variance explained by the specific factor $(A V E S F)$ and the general factor ( $A V E_{-} G F$ ) and the coefficient $H$ of the specific factor $\left(H_{\mathrm{SF}}\right)$ and the general factor $\left(H_{\mathrm{GF}}\right)$. Values between .30 and .60 in the $\omega_{\mathrm{h}}, M E C V_{-} I_{\text {and }} E C V$ reflect a significant and balanced contribution; values below .30 indicate a poor contribution and values above .70 an excessive contribution (Reise et al., 2018). Considering a minimum AVE of .50 and a contribution of at least $30 \%$, the minimum value of the $A V E$ for the general or specific factor should be .15 . The maximum value would be .70 for a maximum contribution of $70 \%$ when explaining the entire variance. An $H$ coefficient between $>.47$ (AVE of .15 with homogeneous load of .39) and $\leq .92$ ( $A V E$ of .70 with homogeneous load of .84) show a balanced contribution of the factor (specific or general) with five indicators, between $>.51$ and $\leq .93$ with 6 indicators, between $>.68$ and $\leq .97$ with 12 indicators, and between $>.75$ and $\leq .98$ with 17 indicators (Moral, 2019b).

\section{Ethical considerations}

The names of those responsible for the study and their electronic addresses were provided so that the participants could request information or support in relation to any question raised by the study. No identification data were asked for to the participants in order to assure their anonymity, and the confidentiality of the information provided through this questionnaire was guaranteed.

\section{RESULTS}

The sample was composed of 600 health science students from three first years of carrier. The sample from the first university was, $n=300$ out of $N=450$, sample proportion, $s p$ $=.67$. From the second university, $n=300$ out of $N=2000$, $s p=.15$. Regarding sex, $57.3 \%(n=344)$ were women and $42.7 \%(n=256)$ were men. The mean age ranged from 17 to 21 years $(M=20.24, S D=2.56)$, and it was not statistically equivalent between both sexes $\left(t_{[598]}=.83, p=.41\right)$. There were not lost or excluded cases in the sample.
Table 1

Composite and construct reliability, and convergent and discriminant validity in the five models for SWB

\begin{tabular}{lcccccc}
\hline & & \multicolumn{2}{c}{ Reliability } & $\begin{array}{c}\text { Convergent } \\
\text { validity }\end{array}$ & \multicolumn{2}{c}{ Discriminant validity } \\
\cline { 3 - 8 } Model & Factor & $\omega$ & $H$ & AVE & Factors & $r^{2}$ \\
\hline $1 \mathrm{~F}$ & SWB & .921 & .943 & .421 & & \\
\multirow{2}{*}{$2 \mathrm{CF}$} & Affect & .922 & .939 & .505 & Affect and LS & .342 \\
& LS & .859 & .884 & .552 & & \\
& PA & .870 & .903 & .539 & PA and NA & .473 \\
$3 \mathrm{CF}$ & NA & .929 & .932 & .685 & PA and LS & .355 \\
& LS & .859 & .883 & .553 & NA and LS & .171 \\
\hline
\end{tabular}

Notes: Models: $1 \mathrm{~F}=$ One factor, $2 \mathrm{CF}=$ Two Correlated Factors, and 3CF = Three Correlated Factors. Factors: SWB $=$ Subjective Well-Being, LS $=$ Life Satisfaction, PA = Positive Affect, and NA = Negative Affect. Statistics: $\omega=$ McDonald's composite reliability coefficient, $\mathrm{H}=$ Hancock-Muller's construct reliability coefficient, $\mathrm{AVE}=$ Average Variance Explained, and $r^{2}=$ shared variance between factors.

Five recursive models with independent measurement residuals were specified. In the one-factor model (1F); all parameters were significant, its parsimony was very high, and showed excellent levels of composite and construct reliability coefficients (Table 1). However, the $A V E$ was below .50 $(A V E=.42)$ and its fit to the data was bad through the eight indices (Table 2). Therefore, it is a bad model due to the lack of convergent validity and poor fit to data.

In the model with two correlated factors (2CF), the items composing SPANE were determined by an affect factor and the items composing SWLS by a LS factor. All the parameters were significant. Both factors showed convergent and discriminant validity. The reliability of the affect factor was excellent and the one for the LS factor was good (Table 1). The goodness of fit improved with respect to the one-factor model $\left(\Delta \chi^{2}[\Delta d f=1]=816.37, p<.001, \Delta \chi^{2} / \Delta d f=816.37\right.$, $\Delta N F I=.13, \Delta N N F I=.15$, and $\triangle C F I=.13)$, but it was bad by seven indices and acceptable for SRMR (Table 2). The parsimony was also very high and the relationship between adjustment and parsimony was acceptable (Table 2). Therefore, the great weakness of the model was its fit to data.

In the bifactor model with two specific factors (BF_2SF), the items composing SPANE were determined by a SWB general factor and an affect specific factor; in turn, the items composing SWLS were determined by a SWB general factor and a LS specific factor. The two specific factors were independent. There were found non-significant parameters: the weights of the affect specific factor on items 1, 5, 7, and 12 that correspond to PA. All other parameters were significant. The $\omega_{\mathrm{h}}, A V E, E C V$ and $M \_E C V \_I$ indices show that the affect specific factor has a poor contribution to its content domain of 12 items; in turn, the $E C V$ and $M_{-} E C V_{-} I$ indices indicated that the general factor has an excessive contribution to these 12 items. The contributions of the specific and the general factors to the content domain of the 5 items composing SWLS were balanced. 
Table 2

Fit indices in the five models for SWB

\begin{tabular}{lccccc}
\hline Fit indices & $1 F$ & $2 C F$ & $B F-2 S F$ & $3 C F$ & $B F-3 S F$ \\
\hline$\chi^{2}$ & 1962.753 & 1146.38 & 281.147 & 369.644 & 255.325 \\
df & 119 & 118 & 102 & 116 & 102 \\
p-value & $<.001$ & $<.001$ & $<.001$ & $<.001$ & $<.001$ \\
$\chi^{2} /$ df & 16.494 & 9.715 & 2.756 & 3.187 & 2.503 \\
GFI & .633 & .747 & .947 & .932 & .953 \\
AGFI & .529 & .672 & .920 & .910 & .929 \\
NFI & .697 & .823 & .957 & .943 & .961 \\
NNFI & .667 & .813 & .962 & .953 & .968 \\
CFI & .709 & .838 & .972 & .960 & .976 \\
RMSEA & .161 & .121 & .054 & .060 & .050 \\
[90\% CI] & {$[.155, .167]$} & {$[.114, .127]$} & {$[.047, .062]$} & {$[.054, .067]$} & {$[.042, .058]$} \\
p-Close & $<.001$ & $<.001$ & .176 & .007 & .479 \\
1- $\beta$ & 1 & 1 & .219 & .756 & .052 \\
SRMR & .114 & .077 & .028 & .039 & .032 \\
PR & .875 & .868 & .750 & .853 & .750 \\
PNFI & .610 & .714 & .717 & .804 & .720 \\
PNNFI & .584 & .706 & .722 & .813 & .726 \\
PCFI & .620 & .727 & .729 & .819 & .732 \\
PGFI & .493 & .576 & .631 & .706 & .635 \\
\hline
\end{tabular}

Notes: Fit indices: $\chi^{2}=$ Chi-squared static, $d f=$ degree of freedom, and $p$-value $=$ probability value for a two-tailed test, $\chi^{2} / d f=$ relative chi-square, $G F I=$ Goodness-of-Fit Index, $A G F I=$ Adjusted Goodness-of-Fit Index, NFI = Normed Fit Index, NNFI = Non-Normed Fit Index, CFI $=$ Comparative Fit Index, RMSEA = Root Mean Square Error of Approximation with its point estimate and $90 \%$ confidence interval, p-close = probability value for null hypothesis of close fit $\left(\mathrm{H}_{0}: R M S E A \leq .05\right)$, and $1-\beta=$ power or probability of rejecting the null hypothesis $\left(\mathrm{H}_{0}: R M S E A\right.$ $\leq .05)$, when it is false, $P R=$ Parsimony Ratio, $P N F I=$ Parsimonious Normed Fit Index, $P N N F I$ $=$ Parsimonious Non-Normed Fit Index, $P C F I=$ Parsimonious Comparative Fit Index, and $P G F I=$ Parsimonious Goodness-of-Fit Index. Models: $1 \mathrm{~F}=$ one factor, $2 \mathrm{CF}=$ Two Correlated Factors, BF_2SF = Bifactor Model with Two Specific Factors, 3CF = Three Correlated Factors, and BF_3SF $=$ Bifactor Model with Three Specific Factors.

However, the contribution of the two specific factors to the full set of 17 items did not show reliability through $\omega_{\mathrm{h}}(\mathrm{Ta}-$ ble 3). The goodness of fit was good through six indices and acceptable through $\chi^{2} / d f$ and GFI (Table 2), and improved

Table 3

Indices of internal consistency reliability and convergent validity

\begin{tabular}{|c|c|c|c|c|c|c|c|c|c|c|c|}
\hline \multirow[b]{2}{*}{ Content domain } & \multirow{2}{*}{$\begin{array}{l}\text { Source } \\
\text { of effect }\end{array}$} & \multicolumn{2}{|c|}{$\omega_{h}$} & \multicolumn{2}{|c|}{$H$} & \multicolumn{2}{|c|}{ AVE } & \multicolumn{2}{|c|}{$E C V$} & \multicolumn{2}{|c|}{ M_ECV_I } \\
\hline & & $2 S F$ & $3 S F$ & $2 S F$ & $3 S F$ & $2 S F$ & $3 S F$ & $2 S F$ & $3 S F$ & $2 S F$ & $3 S F$ \\
\hline \multirow[t]{3}{*}{ Affect or NA } & Pooled & .528 & .468 & .960 & .907 & .617 & .545 & & & & \\
\hline & SF & .090 & .289 & .716 & .690 & 141 & .254 & .229 & .466 & .261 & .476 \\
\hline & GF & .411 & .315 & .942 & .732 & .476 & .291 & .771 & .534 & .739 & .524 \\
\hline \multirow[t]{3}{*}{ PA } & Pooled & & .493 & & .943 & & .717 & & & & \\
\hline & SF & & .081 & & .391 & & .092 & & .129 & & .131 \\
\hline & GF & & .449 & & .919 & & .625 & & .871 & & .869 \\
\hline \multirow[t]{3}{*}{ LS } & Pooled & .464 & .464 & .883 & .883 & .557 & .558 & & & & \\
\hline & SF & .359 & .361 & .750 & .754 & .363 & .367 & .652 & .658 & .652 & .657 \\
\hline & GF & .229 & .226 & .553 & .548 & .194 & .191 & .348 & .342 & .348 & .343 \\
\hline \multirow[t]{3}{*}{ SWB } & Pooled & .606 & .512 & .969 & .971 & .599 & .609 & & & & \\
\hline & SF & $<.001$ & .240 & .847 & .856 & .206 & .230 & .345 & .378 & .376 & .407 \\
\hline & GF & .369 & .360 & .946 & .938 & .393 & .379 & .655 & .622 & .624 & .593 \\
\hline
\end{tabular}

Notes: Content domain: NA = Negative Affect, PA = Positive Affect, LS = Satisfaction with Life, and SWB = Subjective Well-Being. Source of effect: Pooled effect of both factors, SF = effect of specific factor, and GF = effect of general factor (SWB). Indices: $\omega_{\mathrm{h}}=\mathrm{McDonald}$ 's hierarchical omega, $H=$ Hancock-Muller's coefficient $H, A V E=$ Average Variance Extracted, $E C V=$ Explained Common Variance, $M \_E C V \_I=$ Mean of Explained Common variance for item. 
Table 4

Model comparison in goodness of fit

\begin{tabular}{|c|c|c|c|c|c|c|c|c|}
\hline \multirow[b]{2}{*}{ Difference indices } & \multicolumn{4}{|c|}{ BF-3SF versus } & \multicolumn{4}{|c|}{$3 C F$ versus } \\
\hline & $1 F$ & $2 C F$ & $B F-2 S F$ & $3 C F$ & $1 F$ & $2 C F$ & $B F-2 S F$ & $B F-3 S F$ \\
\hline$\Delta \chi^{2}$ & 1707.428 & 891.055 & 25.822 & 114.319 & 1593.109 & 776.736 & 88.497 & 114.319 \\
\hline$\Delta d f$ & 17 & 16 & 0 & 14 & 3 & 2 & 14 & 14 \\
\hline$p$-value & $<.001$ & $<.001$ & - & $<.001$ & $<.001$ & $<.001$ & $<.001$ & $<.001$ \\
\hline$\Delta \chi^{2} / \Delta d f$ & 10.437 & 55.691 & - & 8.166 & 531.036 & 388.368 & 6.321 & 8.166 \\
\hline$\Delta N F I$ & .264 & .138 & .004 & .018 & .246 & .120 & .014 & .018 \\
\hline$\triangle N N F I$ & .301 & .155 & .006 & .015 & .286 & .14 & .009 & .015 \\
\hline$\triangle C F I$ & .267 & .138 & .004 & .016 & .251 & .122 & .012 & .016 \\
\hline
\end{tabular}

Notes: Difference indices: $\Delta \chi^{2}=$ the chi-square difference test, $\Delta d f=$ degree of freedom difference, $p$-value = probability value for a two-tailed test, $\Delta \chi^{2} / \Delta d f=$ Relative Chi-Square Difference, $\Delta N F I=$ Normed Fit Index difference, $\Delta N N F I=$ Non-Normed Fit Index difference, $\Delta C F I=C o m p a r a t i v e$ Fit Index difference. Models: 1F = one factor, 2CF = Two Correlated Factors, BF_2SF = Bifactor Model with Two Specific Factors, 3CF = Three Correlated Factors, and BF_3SF = Bifactor Model with Three Specific Factors.

with respect to the two previous models (Table 4). The parsimony was high and the parsimonious indices showed an acceptable relation between fit and parsimony (Table 2). The weakness of this model was in the contribution of affect specific factor and in the consistency of the two specific factors within the full set of items.

In the model with three correlated factors $(3 \mathrm{CF})$, items $1,3,5,7,10$, and 12 composing SPANE were determined by a NA factor; items 2, 4, 6, 8, 9, and 11 composing SPANE by a PA factor; and the items composing SWLS by a LS factor (figure 1). All its parameters were significant.
All three factors showed convergent $(A V E>.50)$ and discriminant $\left(r^{2}<A V E\right)$ validity, and a reliability from good to excellent (Table 2). Goodness of fit was good through four indices ( $A G F I, N N F I, C F I$, and SRMR), acceptable through three (GFI, NFI, and RMSEA), and bad through $\chi^{2} / d f$ (Table 4$)$. Thus, the goodness of fit of this model was better than the one showed by the one-factor model and the $2 \mathrm{CF}$ model, but it was worse than the one showed by the BF_2SF (Table 4). The parsimony was very high and four parsimonious indices reflected a good relation between fit and parsimony (Table 3 ). Compared to previous models,

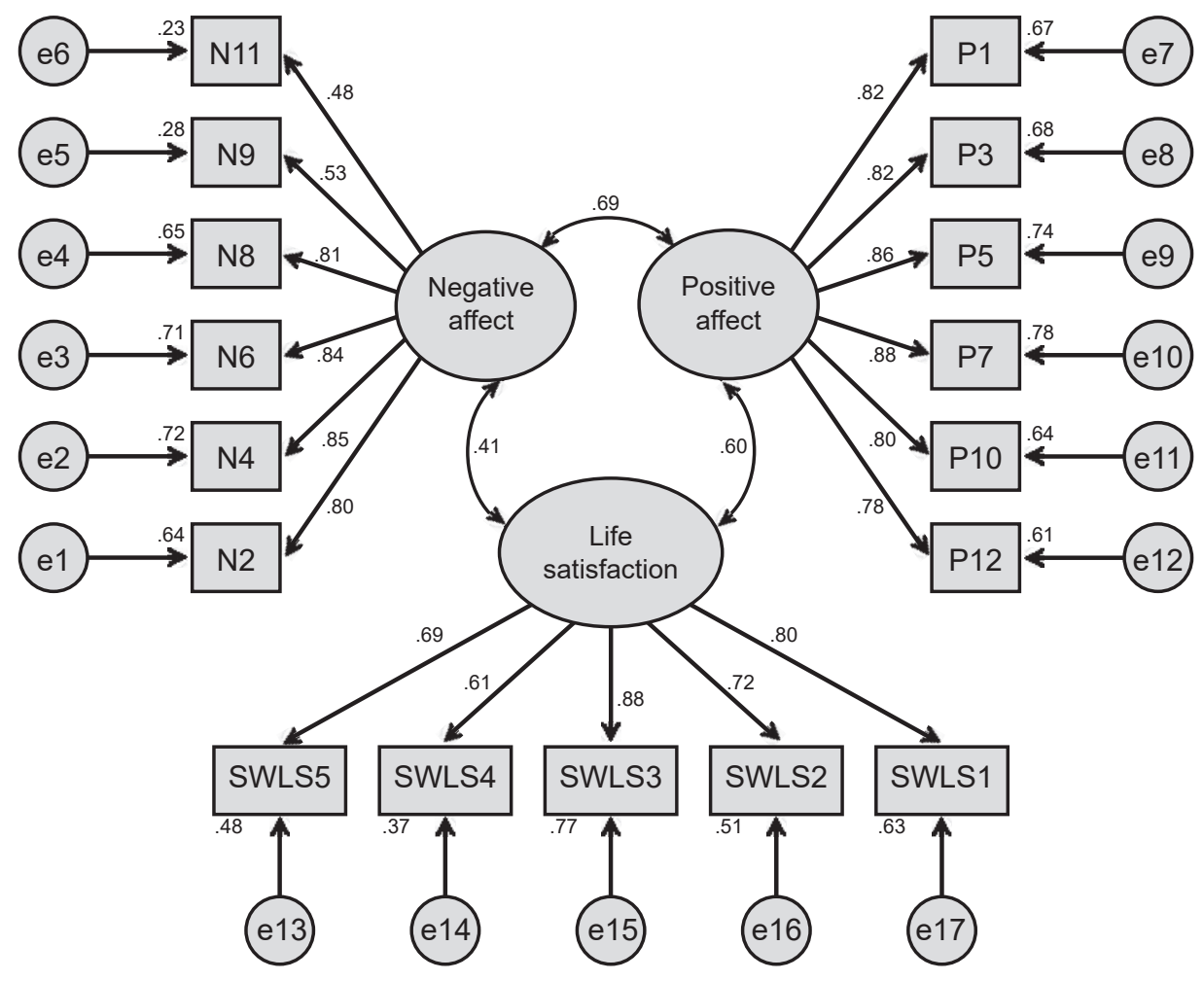

Figure 1. Model composed of three correlates factors for SWB estimated by maximum likelihood method in the sample of 600 health sciences students. 


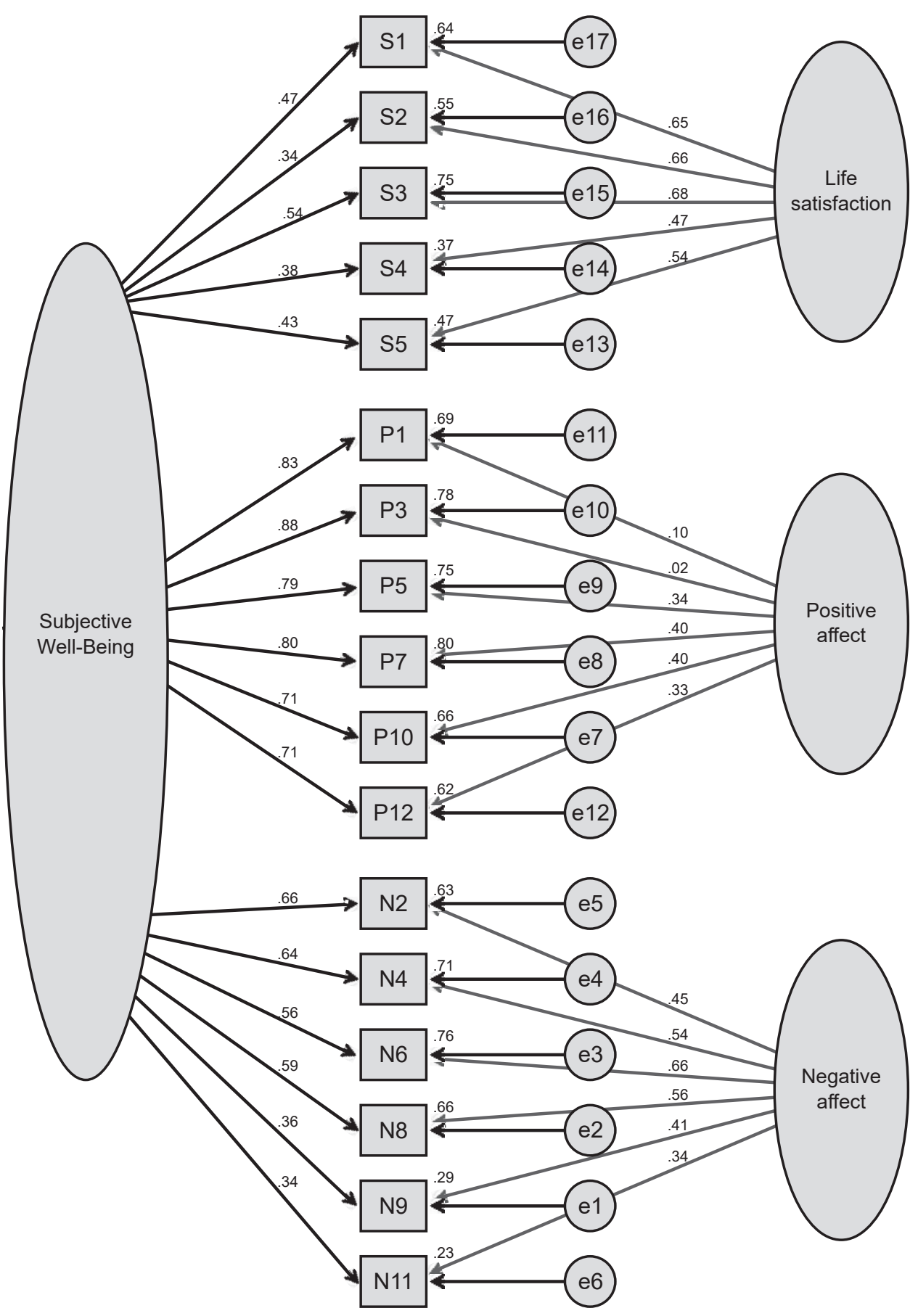

Figure 2. Bifactor model comprising three specific factors for SWB estimated by maximum likelihood method in the sample of 600 health sciences students.

BF_2SF showed better properties, and therefore it is more desirable.

In the bifactor model with three specific factors (BF_3SF), the 17 items were determined by a general factor. Items $1,3,5,7,10$, and 12 composing SPANE were determined by a NA specific factor; items 2, 4, 6, 8, 9, and 11 composing SPANE by a PA specific factor; and the items composing SWLS by a LS specific factor. The three specific factors are independent (Figure 2). The weights of the PA specific factor on items 1 and 3 were not significant. All other parameters were significant. The contribution of the three specific factors and the general factor to the full set of 17 items was balanced by all the indices, as well as the contribution of the specific factor and the general factor to 
the items composing SWLS and to the items related to NA, although the $\omega_{\mathrm{h}}$ of the NA specific factor was slightly below .30. There was also an excessive contribution of the general factor to the items related to PA to the detriment of the specific factor (Table 3). Its goodness of fit was good by seven indices and acceptable by one. The parsimony was high, and its parsimonious fit indices were acceptable (Table 2). Compared with the 1F, 2CF, and 3CF models, BF_3SF showed a better fit (Table 4). Although its fit was equivalent to BF_2SF (Table 4), this model constitutes a better representation of the SWB because the contributions of its general factor and specific factors to the content domains and the full set of items were more balanced (Table 3).

\section{DISCUSSION AND CONCLUSION}

The main objective of this study was to analyze the SWB bifactor model using SPANE to assess affect instead of PANAS. Likewise, from a transcultural perspective, PANAS includes items that could not be properly considered as affects (Diener et al., 2010). Thus, this study provides evidence for the application of the bifactor model as an alternative to other models (Busseri \& Sadava, 2011). It also shows that SPANE offers better results to assess the two specific factors of affect than PANAS (Daniel-González et al., 2019).

In this study, BF_3SF and all its independent residuals had a better fit than the other four models that were specified. Besides, all its goodness-of-fit indices were good, its parsimony was high, and its relation between fit and parsimony was acceptable. Even though there is no evidence of other research in which SPANE has been used to study the structure of SWB, it can be concluded that the present findings coincide with those found by Jovanović (2015), and represent evidence in favor of BF_3SF. Nevertheless, compared to the study conducted by Jovanović (2015), in the present study it was not necessary to correlate residuals in order to obtain a good fit. Precisely, Rush and Hofer (2014) pointed out that, when using PANAS, it is necessary to free parameters of correlation between residuals in order to achieve a good fit. Consequently, the present results support the proposal about using SPANE to assess the affective component, since its content has greater cross-cultural validity than PANAS (Diener et al., 2010).

In this study, the $1 \mathrm{~F}$ and $2 \mathrm{CF}$ models had a poor fit. Therefore, they can be discarded. It should be noted that BF_3SF is comparable to BF_2SF in goodness of fit, but BF_3SF surpasses BF_2SF in convergent validity, discriminant validity, and internal consistency reliability.

Clearly, the present findings support the existence of a SWB general factor, but not a one-factor model. In BF3SF, almost two thirds of the common variance explained of SWB is attributable to general factor, and just over a third to the three specific factors. The aforementioned statement indicates a substantive contribution of both the general and specific factors to the SWB. The same happens in the domains of LS and NA. However, there is an excessive contribution of the general factor to the PA domain to the detriment of the specificity of this factor.

It should be noted that, notwithstanding the fact that the goodness of fit of $3 \mathrm{CF}$ was surpassed by BF_3SF, most of the goodness-of-fit indices yielded by $3 \mathrm{CF}$ were good. The 3CF model was more parsimonious than the BF_3SF model, showed a better relation between fit and parsimony, and its factors had convergent, discriminant validity, and internal consistency. From the set of psychometric properties, $3 \mathrm{CF}$ surmounts BF $3 \mathrm{SF}$, although this latter constitutes the best theoretical justification for the calculation of a total SWB score.

The creation of composite scores of SWB has been criticized from the theoretical point of view because they do not take into account the individual character of the two affective components with different valence and the cognitive component of the SWB (Jovanović, 2015; Chen, Bai, Lee, \& Jing, 2016). This research advocates a score composed of three factors. Its strongest theoretical support would come from BF_3SF with a weakness in the contribution of the specific PA factor. In turn, its strongest empirical base would come from $3 \mathrm{CF}$, since there was a significant interrelation of medium to high between the three factors with clearly discernible variances.

Regarding the limitations of this study, first, a non-probabilistic sampling was used, and thus inferences should be taken with due caution and circumscribed to health sciences university students. The present results constitute comparison data and hypotheses for other populations. Second, the study design was non-experimental, hence it is not possible to make causal inferences, and one can only speak in terms of interrelation or structure.

In conclusion, the present data provide empirical support to BF_3SF. SPANE has a good fit without the need to correlate measurement residuals. When considering the full set of properties (goodness of fit, parsimony, fit-parsimony relationship, convergent validity, discriminant validity, and internal consistency reliability), 3CF offers the best result. Both models justify the use of a composite score of SWB and scores for three specific content domains (PA, NA, and LS).

It is suggested to replicate this research in other populations and cultures in order to determine if there are differences or similarities in the structure of SWB, with the added value of the use of SPANE and the various indices of goodness-of-fit, parsimony, convergent validity, divergent validity, and reliability. Most research focuses on goodness of fit and ignores all these aspects of great relevance (Reise et al., 2018). The present investigation did not address the practical importance of the bifactor model of SWB. The future studies can be conducted under the premise of ex- 
amining the components of the bifactor model to predict or explain variables such as academic performance, work performance, and mental health.

\section{Funding}

None.

\section{Conflicts of interest}

The authors declare they have no conflicts of interest.

\section{REFERENCES}

Busseri, M. A. (2015). Toward a resolution of the tripartite structure of subjective well-being. Journal of Personality, 83(4), 413-428. doi: 10.1111/jopy.12116

Busseri, M. A. (2018). Examining the structure of subjective well-being through meta-analysis of the associations among positive affect, negative affect, and life satisfaction. Personality and Individual Differences, 122, 68-71. doi: 10.1016/j. paid.2017.10.003

Busseri, M. A., \& Sadava, W. (2011). A review of the tripartite structure of subjective well-being: Implications for conceptualization, operationalization, analysis, and synthesis. Personality and Social Psychology Review, 15(3), 290-314. doi: $10.1177 / 1088868310391271$

Byrne, B. M. (2016). Structural Equation Modeling with AMOS. Basic Concepts, Applications, and Programming (3rd Ed.). New York: Routledge. doi: $10.4324 / 9781315757421$

Chen, F. F., Bai, L., Lee, J. M., \& Jing, Y. (2016). Culture and the structure of affect: A bifactor modeling approach. Journal of Happiness Studies, 17(5), 1801-1824. doi: 10.1007/s10902-015-9671-3

Chen, F. F., Jing, Y., Hayes, A., \& Lee, J. (2013). Two concepts or two approaches? A bifactor analysis of psychological and subjective well-being. Journal of Happiness Studies, 14(3), 1033-1068. doi: 10.1007/s10902-012-9367-x

Daniel-González, L., Moral de la Rubia, J., Valle de la O, A., García-Cadena, C. H., \& Martínez-Martí, M. L. (2019). Validation of the Mexican spanish version of the scale of positive and negative experience in a sample of medical and psychology students. Psychological Reports, 003329411989604. doi: $10.1177 / 0033294119896046$

Diener, E. (1984). Subjective well-being. Psychological Bulletin, 95(3), 542-575. doi: 10.1037/0033-2909.95.3.542

Diener, E., Emmons, R. A., Larsen, R. J., \& Griffin, S. (1985). The satisfaction with life scale. Journal of Personality Assessment, 49(1), 71-75. doi: 10.1207/ s15327752jpa4901 13

Diener, E., Heintzelman, S. J., Kushlev, K., Tay, L., Wirtz, D., Lutes, L. D., \& Oishi, S. (2017). Findings all psychologists should know from the new science on subjective well-being. Canadian Psychology, 58(2), 87-104. doi: 10.1037/ cap0000063

Diener, E., Lucas, R. E., \& Oishi, S. (2018). Advances and open questions in the science of subjective well-being. Collabra: Psychology, 4(1), 15. doi: 10.1525/ collabra. 115

Diener, E., Wirtz, D., Tov, W., Kim-Prieto, C., Choi, D., Oishi, S., \& Biswas-Diener, R. (2010). New well-being measures: Short scales to assess flourishing and positive and negative feelings. Social Indicators Research, 97(2), 143-156. doi: 10.1007/s11205-009-9493-y
Jovanović, V. (2015). A bifactor model of subjective well-being: A re-examination of the structure of subjective well-being. Personality and Individual Differences, 87, 45-49. doi: 10.1016/j.paid.2015.07.026

Lapuente, L., Dominguez-Lara, S., Flores-Kanter, P. E., \& Medrano, L. A. (2018) Estructura del bienestar subjetivo mediante análisis bifactor: ¿Unidimensional o multidimensional? Avaliação Psicológica, 17(2), 252-259. doi: 10.15689/ ap.2018.1702.14521.11

Maddux, J. E. (2018) Subjective well- being and life satisfaction: An introduction to conceptions, theories, and measures. In Maddux, J. E. (Ed.), Subjective WellBeing and Life Satisfaction (pp 3-31). New York: Routledge.

Metler, S. J., \& Busseri, M. A. (2015). Further evaluation of the tripartite structure of subjective well-being: Evidence from longitudinal and experimental studies. Journal of Personality, 85(2), 192-206. doi: 10.1111/jopy.12233

Moral, J. (2019a). La escala de afectos positivos y negativos (PANAS) en personas mexicanas casadas de Monterrey. Interacciones: Revista de Avances en Psicología, 5(1), 35-50. doi: 10.24016/2019.v5n1.151

Moral, J. (2019b). Revisión de los criterios para validez convergente estimada a través de la Varianza Media Extraída. Psychologia, 13(2), 25-41. doi: $10.21500 / 19002386.4119$

Quezada, L., Landero, R., \& Gonzalez, T. (2016). A validity and reliability study of the subjective happiness scale in Mexico. The Journal of Happiness \& WellBeing, 4(1), 90-100.

Rahm, T., Heise, E., \& Schuldt, M. (2017). Measuring the frequency of emotionsvalidation of the Scale of Positive and Negative Experience (SPANE) in Germany. Plos One, 12(2), e0171288. doi: 10.1371/journal.pone.0171288

Reise, S. P., Bonifay, W., \& Haviland, M. G. (2018). Bifactor modelling and the evaluation of scale scores. In Irwing, P., Booth, T., \& Hughes, D. J. (Eds.), The Wiley Handbook of Psychometric Testing (pp 675-707). doi: 10.1002/9781118489772.ch22

Rodríguez-Fernández, A., \& Goñi-Grandmontagne, A. (2011). La estructura tridimensional del bienestar subjetivo. Anales de Psicología, 27(2), 327-332.

Rush, J., \& Hofer, S. M. (2014). Differences in within-and between-person factor structure of positive and negative affect: Analysis of two intensive measurement studies using multilevel structural equation modeling. Psychological Assessment, 26(2), 462-473. doi: 10.1037/a0035666

Seib-Pfeifer, L. E., Pugnaghi, G., Beauducel, A., \& Leue, A. (2017). On the replication of factor structures of the positive and negative affect schedule (PANAS). Personality and Individual Differences, 107, 201-207. doi: 10.1016/j. paid.2016.11.053

Soper, D. S. (2020). A-priori sample size calculator for structural equation models [Software]. Available from http://www.danielsoper.com/statcalc

Vázquez, C., Duque, A., \& Hervas, G. (2013). Satisfaction with life scale in a representative sample of spanish adults: Validation and normative data. The Spanish Journal of Psychology, 16, e82. doi: 10.1017/sjp.2013.82

Watson, D., Clark, L. A., \& Tellegen, A. (1988). Development and validation of brief measures of positive and negative affect: The PANAS scales. Journal of Personality and Social Psychology, 54(6), 1063-1070. doi: 10.1037/00223514.54.6.1063

Westland, J. C. (2010). Lower bounds on sample size in structural equation modeling. Electronic Commerce Research and Applications, 9(6), 476-487. doi: 10.1016/j. elerap.2010.07.003 